\title{
The effective action and quantum gauge transformations
}

\author{
S. Alexandrov* \\ Department of Theoretical Physics, St.Petersburg University,198904 St.Petersburg, Russia
}

The local symmetry transformations of the quantum effective action for general gauge theory are found. Additional symmetries arise under consideration of background gauges. Together with "trivial" gauge transformations, vanishing on mass shell, they can be used for construction simple gauge generators. For example, for the Yang-Mills theory the classically invariant effective action is obtained, reproducing DeWitt's result. For rank one theories a natural generalization is proposed.

\section{INTRODUCTION}

The concept of symmetry was and remains a very powerful tool for construction of the quantum field theory. One of its main virtues is that the symmetry restricts a form of the action, which lies in the ground of the theory. Consequences of the classical symmetry play a crucial role for renormalizability of the quantum theory. And in the investigation of this problem the effective action takes a very important place [1]. Besides it is the only quantum object in which the symmetry should be reflected by the same way as in the classical action also restricting the number of available structures. So it is natural to find this quantum realization of the symmetry, i.e. the symmetry transformations of the effective action, in an explicit form.

One of first steps in this direction was done by DeWitt in his construction of the classically gauge invariant effective action for the Yang-Mills theory [2]. This work gave rise to the number of papers devoted to this problem [3]. But all of them do not go beyond linear gauge transformations and background gauges of the certain kind. This is a very strong limitation on the physical theory. As we know the Hamiltonian forms of gravity, supersymmetry theories and many others require nonlinear transformations. So an investigation of general gauge theories from the point of view of the quantum gauge symmetry is needed.

The following break-through is connected with the concept of the effective average action or Vilkovisky-DeWitt's action [1] - [6]. Its gauge invariance and gauge independence are very attractive properties. However its actual construction in arbitrary gauge and for arbitrary gauge theory is an enormously hard task because the connection on the frame bundle on the space-of-histories is needed. Besides the effective average action is connected to the ordinary generating functional for the one-particle-irreducible Green functions in a nontrivial way. So we simply bypass the subject and consider the effective action constructed in the usual way as Legendre transformation.

The common approach to the symmetry properties of the effective action for general gauge theories is investigation of the Ward identities (see for example [7], [8]). They are the reflection of the global BRST symmetry which replaces the gauge symmetry in path integral quantization [9]. This symmetry plays a leading role in quantization of general gauge theories being the basis for Hamiltonian BFV 10 and Lagrangian BV [11] quantization schemes. Within these approaches global symmetry transformations of the effective action, which are called quantum BRST transformations, can easily be found 12 15]. Here we are interested in their local counterparts which are realized on the physical fields only. Explicit formulas for them, to our knowledge, are absent in the literature and our aim is to fill in this gap. Besides we discuss the symmetry transformations in presence of background fields and apply the obtained results to the rank one theory.

\footnotetext{
*e.mail: alexand@snoopy.phys.spbu.ru
} 
Our consideration is based on the Hamiltonian form of BRST quantization. An alternative strategy is to follow the BV formalism. Some remarks on this point are given in Sec.VI.

The paper is organized as follows. In Sec.II the BFV quantization is reviewed and for completeness and to fix the notations the quantum BRST transformations are obtained. In the subsequent section the gauge transformations of the effective action in terms of quantum averages are found and the "trivial" transformations and the symmetry algebra are discussed. In Sec.IV it is shown that introduction of background fields results in appearance of additional symmetries which can be combined with initial ones. This is used in Sec.V to construct the symmetry, which is reduced to the classical gauge transformations in the case of the Yang-Mills theory reproducing DeWitt's result and gives a generalization for nonlinear transformations. In the last section some problems and perspectives are outlined.

Our condensed notations correspond to DeWitt's ones introduced in [16]. This may lead to confusion when they are applied, for example, in an expression for the classical action. In such cases all tensors must be understood as local what results in locality of the whole expression. In this connection it is convenient to extend the definition of the Poisson brackets on variables depending on different moments of time. We demand $\left\{q^{s}, p_{r}\right\}=\delta_{r}^{s}$, i.e. an unsimultaneous commutator vanishes. This provides locality and keeps unity of the notations. All derivatives with respect to Grassmann variables are left and for simplicity we restrict ourselves to the case of even classical fields only.

\section{PRELIMINARIES}

Let us survey the Hamiltonian BFV quantization following the review 17].

Consider a gauge theory with phase space variables $z^{A}=\left(q^{s}, p_{s}\right)$, Hamiltonian $H_{0}(q, p)$ and first class constraints $G_{\alpha}$. Let $n^{\alpha}$ be the Lagrange multipliers associated with the constraints $G_{\alpha}$, and $\pi_{\alpha}$ be the canonically conjugate momenta. The action is given by

$$
S(q, p, n)=\dot{q}^{s} p_{s}-H_{0}-n^{\alpha} G_{\alpha},
$$

whereas the gauge transformations are $\delta \varphi^{a}=G_{\alpha}^{a}(\varphi) \varepsilon^{\alpha}$, where $\varphi^{a}=\left(q^{s}, p_{s}, n^{\alpha}\right)$ and

$$
G_{\alpha}^{\left(z^{A}\right)}=\left\{z^{A}, G_{\alpha}\right\}, \quad G_{\alpha}^{\left(n^{\beta}\right)}=\delta_{\alpha}^{\beta} \partial_{t}+n^{\gamma} C_{\alpha \gamma}^{\beta}-V_{\alpha}^{\beta}
$$

with $C_{\alpha \beta}^{\gamma}, V_{\alpha}^{\beta}$ defined through the relations $\left\{G_{\alpha}, G_{\beta}\right\}=C_{\alpha \beta}^{\gamma} G_{\gamma}$ and $\left\{H_{0}, G_{\alpha}\right\}=V_{\alpha}^{\beta} G_{\beta}$. The extended phase space is defined by introducing extra ghost and antighost fields $\left(b^{\alpha}, \bar{c}_{\alpha}, c^{\alpha}, \bar{b}_{\alpha}\right)$. obeying the following nonvanishing antibrackets

$$
\left\{b^{\alpha}, \bar{c}_{\beta}\right\}_{+}=-\delta_{\beta}^{\alpha},\left\{c^{\alpha}, \bar{b}_{\beta}\right\}_{+}=-\delta_{\beta}^{\alpha} .
$$

$c^{\alpha}, \bar{c}_{\alpha}$ are real, whereas $b^{\alpha}, \bar{b}_{\alpha}$ are imaginary. It is convenient to define an additional structure on the extended phase space, that of "ghost number". This is done by attributing the following ghost number to the canonical variables: $c^{\alpha}, b^{\alpha}$ have ghost number one, $\bar{c}_{\alpha}, \bar{b}_{\alpha}$ have ghost number minus one. All other variables have ghost number zero.

On this space one can construct a BRST generator $\Omega$ and a BRST invariant Hamiltonian $H$. They are determined by the following conditions:

(a) $\Omega$ is real and odd; (b) $\Omega$ has ghost number one; (c) $\Omega=-i b^{\alpha} \pi_{\alpha}+c^{\alpha} G_{\alpha}+$ "higher ghost terms"; (d) $\{\Omega, \Omega\}_{+}=0$

(a) $H$ is real and even; (b) $H$ has ghost number zero; (c) $H$ coincides with $H_{0}$ up to higher ghost terms; (d) $\{H, \Omega\}=0$

The BRST generator is fully defined by structure functions of the constraint algebra:

$$
\Omega=-i b^{\alpha} \pi_{\alpha}+\sum_{n \geq 0} c^{\alpha_{n+1}} \cdots c^{\alpha_{1}} U_{\alpha_{1} \cdots \alpha_{n+1}}^{(n) \beta_{1} \cdots \beta_{n}} \bar{b}_{\beta_{n}} \cdots \bar{b}_{\beta_{1}} .
$$


For $n=0$ and $n=1$ they are $U_{\alpha}^{(0)}=G_{\alpha}, U_{\alpha \beta}^{(1) \gamma}=-\frac{1}{2} C_{\alpha \beta}^{\gamma}$. Higher order structure functions are defined through repeated Poisson brackets of the constraints. The Hamiltonian $H$ in the first orders in ghosts has the form

$$
H=H_{0}+c^{\alpha} V_{\alpha}^{\beta} \bar{b}_{\beta}+\cdots
$$

The quantization is based on the generating functional for the Green functions which is represented in the form

$$
Z[J]=e^{\frac{i}{\hbar} W(J)}=\int \mathcal{D} \psi^{\prime} e^{\frac{i}{\hbar}\left(S_{e f f}\left(\psi^{\prime}\right)+J_{i} \psi^{\prime i}\right)},
$$

where

$$
S_{e f f}=\dot{q}^{s} p_{s}+\dot{n}^{\alpha} \pi_{\alpha}+\dot{c}^{\alpha} \bar{b}_{\alpha}+\dot{b}^{\alpha} \bar{c}_{\alpha}-H_{e f f}, \quad H_{e f f}=H-\{\Psi, \Omega\}_{+} .
$$

Here $\Psi$ is an odd and imaginary function which has ghost number minus one and plays a role of gauge fixing function, $\psi=(\varphi, \pi, \eta=(c, b), \bar{\eta}=(\bar{c}, \bar{b})) . \varphi^{a}$ are just the physical fields of the theory. From properties of $H$ and $\Omega$ the invariance under the global BRST transformations follows: $\left\{S_{\text {eff }}, \Omega\right\}=$ $-\int d t \frac{d}{d t} \Omega=0$.

We choose

$$
\Psi=\bar{b}_{\alpha} n^{\alpha}+i \bar{c}_{\alpha} \chi^{\alpha}
$$

Substituting (3) and (7) in (6) one obtains [15]:

$$
\begin{aligned}
S_{e f f}= & \dot{q}^{s} p_{s}+\dot{n}^{\alpha} \pi_{\alpha}+\dot{c}^{\alpha} \bar{b}_{\alpha}+\dot{b}^{\alpha} \bar{c}_{\alpha}-H-\sum_{n \geq 0}(n+1) n^{\alpha_{n+1}} c^{\alpha_{n}} \cdots c^{\alpha_{1}} U_{\alpha_{1} \cdots \alpha_{n+1}}^{(n) \beta_{1} \cdots \beta_{n}} \bar{b}_{\beta_{n}} \cdots \bar{b}_{\beta_{1}} \\
& -\pi_{\alpha} \chi^{\alpha}+i b^{\alpha} \bar{b}_{\alpha}-b^{\alpha} \frac{\delta \chi^{\beta}}{\delta n^{\alpha}} \bar{c}_{\beta}+i \sum_{n \geq 0} c^{\alpha_{n+1}} \cdots c^{\alpha_{1}}\left\{U_{\alpha_{1} \cdots \alpha_{n+1}}^{(n) \beta_{1} \cdots \beta_{n}}, \chi^{\gamma}\right\} \bar{b}_{\beta_{n}} \cdots \bar{b}_{\beta_{1}} \bar{c}_{\gamma} .
\end{aligned}
$$

This action gives rise to the BRST extended effective action

$$
\Gamma_{e f f}(\psi)=W(J)-J_{i} \psi^{i}, \quad \frac{\delta W(J)}{\delta J_{i}}=\psi^{i}(J) .
$$

Let us obtain its symmetry transformations. (Following results can be found for instance in [15].) The condition that some transformation is a symmetry of the effective action can be written as

$$
\frac{\delta \Gamma_{e f f}(\psi)}{\delta \psi^{i}} \delta \psi^{i}=-J_{i} \delta \psi^{i}=0 .
$$

Thus the Ward identities homogeneous on the sources are needed. They are immediately obtained from the path integral (5). Due to the BRST invariance of the action $S_{\text {eff }}$ the change of variables $\psi^{\prime i} \rightarrow \psi^{\prime i}+\Omega^{i} \varepsilon$, where $\Omega^{i}(\psi)=\left\{\psi^{i}, \Omega\right\}$, in the first order in $\varepsilon$ leads to the equality

$$
\left.J_{i} \Omega^{i}(\psi)\right|_{\psi \rightarrow \frac{\hbar \delta}{i \delta J}} Z(J)=0 \Leftrightarrow J_{i} \Lambda^{i}=0,
$$

where

$$
\Lambda^{i}(\psi)=\left.e^{-\frac{i}{\hbar} W(J)} \Omega^{i}(\psi)\right|_{\psi \rightarrow \frac{\hbar \delta}{i \delta J}} e^{\frac{i}{\hbar} W(J)} .
$$

The functional $\Lambda^{i}(\psi)$ is just the quantum BRST transformation of the effective action. This result means that the quantum transformation is a vacuum average of $\mathrm{T}$-product of the classical one in the external field, i.e. 


$$
\delta^{(q)} \psi^{i}=\left\langle T\left\{\left.\delta \psi^{i}\right|_{\psi=\hat{\psi}}\right\}\right\rangle_{J(\psi)} .
$$

(This result holds also in the BV formalism [14,18].) In this connection it is convenient to introduce the following notation $\langle f(\psi)\rangle=\langle T\{f(\hat{\psi})\}\rangle_{J(\psi)}$. Then one can write the simple equality

$$
\Lambda^{i}(\psi)=\left\langle\Omega^{i}(\psi)\right\rangle
$$

It is useful to emphasize that quantum corrections to the classical transformation arise from second and higher derivatives of $W(J)$.

\section{THE QUANTUM GAUGE TRANSFORMATIONS}

In the previous section we have obtained the global quantum symmetry of the effective action. To find corresponding local transformations it is necessary to return to the physical fields at quantum level.

It is easy to see (see for example [15]) that with the choice (7) the following conditions reduce (8) to (1)

$$
\eta=\bar{\eta}=0, \quad \pi=0 .
$$

The gauge transformations (2) are restored with help of $\delta \varphi^{a}=\frac{\delta}{\delta c^{\alpha}}\left\{\varphi^{a}, \Omega\right\} \varepsilon^{\alpha}$, the conditions (15) and $\frac{\delta S_{e f f}}{\delta b_{\alpha}}=0$. (The later condition must be used before differentiation over $c^{\alpha}$. Without it there is only the weak invariance under independent transformations generated by $G_{\alpha}$ and $\pi_{\alpha}$.)

Having in mind this classical situation one can impose the conditions (15) on the average fields to extract the gauge invariant effective action. Define

$$
\Gamma=\left.\Gamma_{e f f}\right|_{\substack{\begin{subarray}{c}{n=\bar{\eta}=0 \\
\pi=0} }} \\
{i}\end{subarray}} ^{i} \quad \Lambda_{(0)}^{i}=\left[\Lambda^{i}\right]_{l g}
$$

where the subscript "lg" means a linear on ghost part. From (3) and (14) one obtains the explicit expressions:

$$
\begin{aligned}
\Lambda_{(0)}^{(\eta)} & =\left\langle\{\eta, \Omega\}_{+}\right\rangle_{l g}=0, \Lambda_{(0)}^{\left(\bar{c}_{\alpha}\right)}=\left\langle\left\{\bar{c}_{\alpha}, \Omega\right\}_{+}\right\rangle_{l g}=i \pi_{\alpha}, \\
\Lambda_{(0)}^{\left(\pi_{\alpha}\right)} & =\left\langle\left\{\pi_{\alpha}, \Omega\right\}\right\rangle_{l g}=0, \Lambda_{(0)}^{\left(n^{\alpha}\right)}=\left\langle\left\{n^{\alpha}, \Omega\right\}\right\rangle_{l g}=-i b^{\alpha} .
\end{aligned}
$$

The first equality is obtained due to conservation of the ghost number. Then the "lg" part of (11) with the condition $\pi=0$ yields

$$
\frac{\delta \Gamma(\varphi)}{\delta \varphi^{a}} \Lambda_{(0)}^{\left(\varphi^{a}\right)}=\left[J_{(\bar{b})}^{\alpha}\right]_{l g} \Lambda_{(0)}^{\left(\bar{b}_{\alpha}\right)}
$$

Evidently, $\left[J_{(\bar{b})}^{\alpha}\right]_{l g}=-\left.\frac{\delta^{2} \Gamma_{e f f}}{\delta c^{\beta} \delta b_{\alpha}}\right|_{\eta=\bar{\eta}=0} c^{\beta}-\left.\frac{\delta^{2} \Gamma_{e f f}}{\delta b^{\beta} \delta b_{\alpha}}\right|_{\eta=\bar{\eta}=0} b^{\beta}$. So the condition $\left[J_{(\bar{b})}^{\alpha}\right]_{l g}=0$ can be viewed as a connection of the parameters of local gauge transformations the role of which is played by ghosts $c^{\alpha}$ and $b^{\alpha}$. From (18) these transformations are found to be

$$
\begin{aligned}
& \delta_{c}^{(1)} z^{A}=\Lambda_{(1) \alpha}^{\left(z^{A}\right)} c^{\alpha}, \delta_{c}^{(1)} n^{\alpha}=0, \\
& \delta_{b}^{(2)} z^{A}=\Lambda_{(2) \alpha}^{\left(z^{A}\right)} b^{\alpha}, \delta_{b}^{(2)} n^{\alpha}=-i b^{\alpha},
\end{aligned}
$$

where

$$
\Lambda_{(1) \alpha}^{\left(z^{A}\right)}=\left.\frac{\delta}{\delta c^{\alpha}}\left\langle\left\{z^{A}, \Omega\right\}\right\rangle\right|_{\substack{\eta=\bar{\eta}=0 \\ \pi=0}}, \quad \Lambda_{(2) \alpha}^{\left(z^{A}\right)}=\left.\frac{\delta}{\delta b^{\alpha}}\left\langle\left\{z^{A}, \Omega\right\}\right\rangle\right|_{\substack{\eta=\bar{\eta}=0 \\ \pi=0}} .
$$


Thus, provided $\left[J_{(\bar{b})}^{\alpha}\right]_{l g}=0 \Rightarrow b^{\alpha}=b^{\alpha}(c), \Gamma(\varphi)$ is the effective action invariant under the following quantum gauge transformations

$$
\delta^{(q)} \varphi^{a}=\delta_{\varepsilon}^{(1)} \varphi^{a}+\delta_{b(\varepsilon)}^{(2)} \varphi^{a}=Q_{\alpha}^{a}(\varphi) \varepsilon^{\alpha} .
$$

The closed expression for $Q_{\alpha}^{a}$ can be obtained from (19), (20), (3) and the expressions for ghost propagators.

The analogy of the above mentioned weak invariance (i.e. the invariance under the transformations (19)) comes again from (18). For existing of this symmetry $\Lambda_{(0)}^{\left(\bar{b}_{\alpha}\right)}$ should vanish. This can be done by demanding

$$
\frac{\delta \Gamma}{\delta n^{\alpha}}+i \frac{\delta \Gamma}{\delta z^{A}} \Lambda_{(2) \alpha}^{\left(z^{A}\right)}=0 .
$$

The left-hand side of (22) is an analogy of the classical constraints $G_{\alpha}$ and (22) should be interpreted as a weak equality. Note that in general case the contribution of $q$ and $p$ to this expression does not vanish. So in some sense there is a mixing between the phase space variables $q^{s}, p_{s}$ and the Lagrange multipliers $n^{\alpha}$ due to quantum effects.

Apart from the quantum gauge transformations (21) one can obtain other symmetry transformations of the effective action. Let $j_{a} \equiv J_{\left(\varphi^{a}\right)}$ are sources for the physical fields. Act by the operator $\hat{T}_{\alpha, \ldots}^{\beta, \ldots}=\left.T_{\alpha, \ldots}^{\beta, \ldots}(\varphi, \pi)\right|_{(\varphi, \pi) \rightarrow\left(\frac{\hbar \delta}{i \delta j}, \frac{\hbar \delta}{i \delta J_{(\pi)}}\right)}$ on the identity (11), take the "lg" part and impose the conditions $\pi=0$ and $\left[J_{(\bar{b})}^{\alpha}\right]_{l g}=0$. Then we come to the following equation

$$
j_{a} \frac{\delta}{\delta c^{\lambda}}\left(\hat{T}_{\alpha, \ldots}^{\beta, \ldots} \Lambda_{(0)}^{\left(\varphi^{a}\right)}\right)=i \hbar \frac{\delta}{\delta c^{\lambda}}\left(\frac{\delta \hat{T}_{\alpha, \ldots}^{\beta, \ldots}}{\delta \varphi^{a}} \Lambda_{(0)}^{\left(\varphi^{a}\right)}\right)-i \frac{\delta\left[J_{(\bar{c})}^{\gamma}\right]_{l g}}{\delta c^{\lambda}} \hat{T}_{\alpha, \ldots}^{\beta, \ldots} \pi_{\gamma}(j) .
$$

The demand that the contraction of the right-hand side of (23) with some matrix $M_{\beta}^{\lambda}$ vanishes gives rise to new symmetry transformations:

$$
\delta \varphi^{a}=M_{\beta}^{\lambda} \frac{\delta}{\delta c^{\lambda}}\left(\hat{T}_{\alpha, \ldots}^{\beta, \ldots} \Lambda_{(0)}^{\left(\varphi^{a}\right)}\right) \varepsilon^{\alpha}=\tilde{Q}_{\alpha}^{a}(\varphi) \varepsilon^{\alpha} .
$$

Note that we cannot reject the last term in (23) because $\pi$ is differentiated and the result is not proportional to it. (Vanishing of $\pi$ makes sources dependent and cannot be done before differentiation.) Having in mind this remark one can clarify the sense of these additional transformations. If the condition $\pi=0$ is not hardly used for making the right-hand side of (23) zero differentiation with respect to $j_{a}$ gives $\tilde{Q}_{\alpha}^{a}=-j_{b} \frac{\delta \tilde{Q}_{\alpha}^{b}}{\delta j_{a}}$. Thus new transformations are "trivial" ones vanishing on mass shell. Nevertheless, as we shall see, they play a certain role.

Finally, let us discuss the algebra of the quantum gauge transformations. Here we cannot give any positive result and we are compelled to restrict ourselves to general description of the situation. For derivation of the algebra one should consider

$$
Q_{\alpha}^{b} \frac{\delta}{\delta \varphi^{b}} Q_{\beta}^{a}-Q_{\beta}^{b} \frac{\delta}{\delta \varphi^{b}} Q_{\alpha}^{a},
$$

where $Q_{\alpha}^{a}$ is some quantum average. On the other hand to extract the classical algebra, which is first approximation for the quantum algebra, it is necessary to write down the expression (25) as one quantum average. It can be done but the average of the classical commutator will be only one of many arising terms. So the quantum algebra will be a deformation of the classical one. However in contrast with the usual deformed symmetries, where the algebra is closed in its enveloping algebra, there are additional "trivial" symmetries which can contribute to (25). Of course, on mass shell they must disappear. The situation is similar for the quantum BRST transformation. There is no reason for it to be nilpotent. 


\section{BACKGROUND GAUGES}

Now we introduce in the formalism background fields. It can be done in two ways. First, one can split the quantum fields $\psi$ into the classical part $\mathcal{F}$ and quantum fluctuations. Second, one can take the gauge fixing function dependent on them. We shall not go by the first way for the following reason. It implies that quantum fluctuations are small in some sense and one can demand their vanishing as an invariant condition under gauge transformations. As a result the gauge invariant effective action depending only on background fields can be obtained. In our case however after subtraction of the classical part from an average field the result will not be transformed homogeneously under the quantum gauge transformations and the onefield construction fails.

Thus we work with the full average fields $\psi$ and dependence on the background fields $\mathcal{F}$ comes only from the function $\Psi$. The effective action is a functional of variables of two types, one of them playing a role of external parameters. Since the above-stated considerations don't depend on presence of such parameters the effective action is still invariant under (14) or (21).

It is proved that the presence of background fields gives rise to a new local symmetry including their transformations. Namely, any variation of the background fields can be compensated by an appropriate transformation of the average fields. Indeed, a variation $\delta \mathcal{F}$ leads to

$$
\delta W(J, \mathcal{F})=\frac{i}{\hbar}\left\langle\{\delta \Psi(\psi, \mathcal{F}), \Omega\}_{+}\right\rangle .
$$

On the other hand the BRST transformation of integrated variables in $\langle\delta \Psi\rangle$ gives

$$
\langle\{\delta \Psi, \Omega\}\rangle=\frac{i}{\hbar}\left\langle J_{i} \Omega^{i} \delta \Psi\right\rangle .
$$

Comparing these two equality, it is easy to see that the effective action is invariant under the following transformations

$$
\delta \mathcal{F}^{i}=\varepsilon^{i}, \quad \delta \psi^{i}=\frac{i}{\hbar}\left\langle\left\{\psi^{i}, \Omega\right\} \frac{\delta \Psi}{\delta \mathcal{F}^{j}}\right\rangle \epsilon^{j}=\Lambda_{j}^{(\mathcal{F}) i} \varepsilon^{j} .
$$

Now one can return to the physical sector of the theory. For this nullify all ghosts and $\pi$ in the symmetry equation for $\Gamma_{e f f}$. Then one obtains

$$
\frac{\delta \Gamma(\varphi, \mathcal{F})}{\delta \mathcal{F}^{a}}+\frac{\delta \Gamma(\varphi, \mathcal{F})}{\delta \varphi^{b}} Q_{a}^{(\mathcal{F}) b}=0
$$

where $Q_{a}^{(\mathcal{F}) b}=\left.\Lambda_{a}^{(\mathcal{F}) b}\right|_{\substack{\eta=\bar{\eta}=0 \\ \pi=0}}$ and throughout background fields are introduced for the physical fields only. This identity means that the gauge invariant effective action possesses the additional local symmetry

$$
\delta \mathcal{F}^{i}=\varepsilon^{i}, \quad \delta \varphi^{a}=Q_{b}^{(\mathcal{F}) a} \varepsilon^{b} .
$$

As a result we have the set of local symmetries of $\Gamma$ : the quantum gauge transformations (21), the "trivail" symmetries (24) and the transformations induced by background fields (30). Since any their linear combination is also a symmetry transformation, one can try to find such combination which has some standard form, for example, classical one. It is clear that it cannot be achieved without use of background fields. So it is natural to consider the gauge transformations of the following kind

$$
\delta \mathcal{F}^{a}=G_{\alpha}^{a}(\mathcal{F}) \varepsilon^{\alpha}, \delta \varphi^{a}=\left(Q_{b}^{(\mathcal{F}) a} G_{\alpha}^{b}(\mathcal{F})+Q_{\beta}^{a} X_{\alpha}^{\beta}(\varphi, \mathcal{F})+\hat{Q}_{\beta}^{a} Y_{\alpha}^{\beta}(\varphi, \mathcal{F})\right) \varepsilon^{\alpha}=Q_{\alpha}^{(t o t) a} \varepsilon^{\alpha} .
$$

As we shall see it is possible for the case of the Yang-Mills theory to find the coefficients $X_{\alpha}^{\beta}, Y_{\alpha}^{\beta}$ (and the function $\Psi$ ), which reduce (31) to the classical gauge transformation, what reproduces known DeWitt's result in our formalism. For more general rank one theories the natural generalization also is possible. 


\section{RANK ONE THEORY}

let us consider the rank one theory in which the structure functions $U^{(n)}$ vanish for $n \geq 2$ and the expansion of the BRST invariant Hamiltonian contains only two terms (4). Choose the gauge fixing function in the form (7) with

$$
\chi^{\alpha}=\frac{\alpha}{2}\left(\gamma(\mathcal{F})^{-1}\right)^{\alpha \beta} \pi_{\beta}+\partial_{t} n^{\alpha}+\xi^{\alpha}(\varphi, \mathcal{F}) .
$$

Then the equation (8) reads

$$
\begin{aligned}
S_{e f f}= & S(q, p, n)-\frac{\alpha}{2}\left(\gamma^{-1}\right)^{\alpha \beta} \pi_{\alpha} \pi_{\beta}-\pi_{\alpha} \xi^{\alpha}+\dot{c}^{\alpha} \bar{b}_{\alpha}+i b^{\alpha} \bar{b}_{\alpha}-b^{\alpha} \frac{\delta \xi^{\beta}}{\delta n^{\alpha}} \bar{c}_{\beta} \\
& -c^{\alpha} V_{\alpha}^{\beta} \bar{b}_{\beta}+c^{\alpha} n^{\gamma} C_{\alpha \gamma}^{\beta} \bar{b}_{\beta}+i c^{\alpha}\left\{G_{\alpha}, \xi^{\beta}\right\} \bar{c}_{\beta}+\frac{i}{2} c^{\alpha} c^{\beta}\left\{C_{\alpha \beta}^{\gamma}, \xi^{\lambda}\right\} \bar{b}_{\gamma} \bar{c}_{\lambda} .
\end{aligned}
$$

Integration over $b, \bar{b}$ and $\pi$ in (5) gives

$$
\begin{aligned}
Z\left(j, J_{\pi}, J^{\eta}, J_{\eta}\right)= & \int \mathcal{D} \varphi \mathcal{D} \pi \mathcal{D} c \mathcal{D} \bar{c} \exp \left\{\frac { i } { \hbar } \left(S(\varphi)+\frac{1}{2 \alpha} \gamma_{\alpha \beta}\left(\xi^{\alpha}-J_{(\pi)}^{\alpha}\right)\left(\xi^{\beta}-J_{(\pi)}^{\beta}\right)+j^{a} \varphi_{a}\right.\right. \\
& +i \bar{c}_{\beta} F_{\alpha}^{\beta} c^{\alpha}+\left(J_{\alpha}^{(c)}+i J_{\beta}^{(b)}\left(\delta_{\alpha}^{\beta} \partial_{t}+n^{\gamma} C_{\alpha \gamma}^{\beta}-V_{\alpha}^{\beta}\right)\right) c^{\alpha}-\bar{c}_{\beta}\left(J_{(\bar{c})}^{\beta}-i \frac{\delta \xi^{\beta}}{\delta n^{\alpha}} J_{(\bar{b})}^{\alpha}\right) \\
& \left.\left.+i J_{\alpha}^{(b)} J_{(\bar{b})}^{\alpha}+\frac{1}{2} J_{\gamma}^{(b)} \bar{c}_{\lambda}\left\{C_{\alpha \beta}^{\gamma}, \xi^{\lambda}\right\} c^{\alpha} c^{\beta}+\frac{1}{2} \frac{\delta \xi^{\delta}}{\delta n^{\gamma}} \bar{c}_{\delta} \bar{c}_{\lambda}\left\{C_{\alpha \beta}^{\gamma}, \xi^{\lambda}\right\} c^{\alpha} c^{\beta}\right)\right\},
\end{aligned}
$$

where

$$
F_{\alpha}^{\beta}=\left\{\xi^{\beta}, G_{\alpha}\right\}+\frac{\delta \xi^{\beta}}{\delta n^{\gamma}}\left(\delta_{\alpha}^{\gamma} \partial_{t}+n^{\lambda} C_{\alpha \lambda}^{\gamma}-V_{\alpha}^{\gamma}\right)=\frac{\delta \xi^{\beta}}{\delta \varphi^{a}} G_{\alpha}^{a}
$$

and we omit factors dependent only on background fields since they are canceled by normalization.

Impose the conditions on the gauge: $\left\{C_{\alpha \beta}^{\gamma}, \xi^{\lambda}\right\} \frac{\delta \xi^{\delta}}{\delta n^{\gamma}}=0$ and $\frac{\delta \xi^{\alpha}}{\delta n^{\beta}}$ does not depend on $\varphi$. Due to this the term of fourth order in ghosts in (34) disappears and we can find expressions for the quantum gauge transformations without ghosts. Note that from (34) it follows

$$
c^{\alpha}=-i\left\langle\left(F^{-1}\right)_{\beta}^{\alpha}\left(J_{(\bar{c})}^{\beta}-i \frac{\delta \xi^{\beta}}{\delta n^{\gamma}} J_{(\bar{b})}^{\gamma}+\frac{1}{2}\left\{C_{\gamma \delta}^{\lambda}, \xi^{\beta}\right\} c^{\gamma} c^{\delta} J_{\lambda}^{(b)}\right)\right\rangle_{l g}=-i\left[J_{(\bar{c})}^{\beta}-i \frac{\delta \xi^{\beta}}{\delta n^{\gamma}} J_{(\bar{b})}^{\gamma}\right]_{l g}\left\langle\left(F^{-1}\right)_{\beta}^{\alpha}\right\rangle_{0} .
$$

Here we introduced the notation $\left.\langle\cdot\rangle_{0} \equiv\langle\cdot\rangle\right|_{\eta=\bar{\eta}=0}$. With this equality one obtains

$$
\begin{aligned}
\Lambda_{(0)}^{\left(z^{A}\right)}= & \left\langle c^{\alpha}\left\{z^{A}, G_{\alpha}\right\}+\frac{1}{2} c^{\alpha} c^{\beta}\left\{z^{A}, C_{\alpha \beta}^{\gamma}\right\} \bar{b}_{\gamma}\right\rangle_{l g}=-i\left[J_{(\bar{c})}^{\beta}-i \frac{\delta \xi^{\beta}}{\delta n^{\gamma}} J_{(\bar{b})}^{\gamma}\right]_{l g}\left\langle\left(F^{-1}\right)_{\beta}^{\alpha}\left\{z^{A}, G_{\alpha}\right\}\right\rangle_{0} \\
& -\frac{1}{2}\left[\left.e^{-\frac{i}{\hbar} W}\left\{z^{A}, C_{\alpha \beta}^{\gamma}\right\}\right|_{\varphi \rightarrow \frac{\hbar \delta}{i \delta j}} \frac{\hbar \delta}{i \delta J_{(\bar{b})}^{\gamma}}\left(F^{-1}\right)_{\tau}^{\alpha}\left(J_{(\bar{c})}^{\tau}-i \frac{\delta \xi^{\tau}}{\delta n^{\lambda}} J_{(\bar{b})}^{\lambda}\right)\left(F^{-1}\right)_{\sigma}^{\beta}\left(J_{(\bar{c})}^{\sigma}-i \frac{\delta \xi^{\sigma}}{\delta n^{\delta}} J_{(\bar{b})}^{\delta}\right) e^{\frac{i}{\hbar} W}\right]_{l g} \\
= & \left(\left\langle\left(F^{-1}\right)_{\beta}^{\gamma}\left\{z^{A}, G_{\gamma}\right\}\right\rangle_{0}-i \hbar\left\langle\left(F^{-1}\right)_{\beta}^{\gamma}\left\{z^{A}, C_{\gamma \lambda}^{\tau}\right\}\left(F^{-1}\right)_{\sigma}^{\lambda}\right\rangle_{0} \frac{\delta \xi^{\sigma}}{\delta n^{\tau}}\right)\left(\left\langle F^{-1}\right\rangle_{0}^{-1}\right)_{\alpha}^{\beta} c^{\alpha} \\
\Lambda_{(0)}^{\left(n^{\alpha}\right)}= & -i b^{\alpha}=\left\langle\left(\delta_{\beta}^{\alpha} \partial_{t}+n^{\gamma} C_{\beta \gamma}^{\alpha}-V_{\beta}^{\alpha}\right) c^{\beta}\right\rangle_{l g}+\left[J_{(\bar{b})}^{\alpha}-\frac{i}{2}\left\langle\bar{c}_{\lambda}\left\{C_{\beta \gamma}^{\alpha}, \xi^{\lambda}\right\} c^{\beta} c^{\gamma}\right\rangle\right]_{l g} \\
\underset{\left[J_{(\bar{b})}^{\alpha}\right]_{l g}=0}{=} & \left(\left\langle\left(F^{-1}\right)_{\beta}^{\gamma}\left(\delta_{\gamma}^{\alpha} \partial_{t}+n^{\lambda} C_{\gamma \lambda}^{\alpha}-V_{\gamma}^{\alpha}\right)\right\rangle_{0}-i \hbar\left\langle\left(F^{-1}\right)_{\beta}^{\gamma}\left\{C_{\gamma \lambda}^{\alpha}, \xi^{\sigma}\right\}\left(F^{-1}\right)_{\sigma}^{\lambda}\right\rangle_{0}\right)\left(\left\langle F^{-1}\right\rangle_{0}^{-1}\right)_{\tau}^{\beta} c^{\tau}
\end{aligned}
$$


Since the condition $\left[J_{(\bar{b})}^{\alpha}\right]_{l g}=0$ was not used in $(37)$ we have $\Lambda_{(2) \alpha}^{\left(z^{A}\right)}=0$. So in this case there is no a mixing between the Lagrange multipliers and the phase space variables, and the weak invariance of the effective action is guaranteed by the standard equation $\frac{\delta \Gamma}{\delta n^{\alpha}}=0$. This is a direct consequence of the above imposed conditions on the gauge fixing function.

The transformations (37), 38) can be incorporated into one expression:

$$
\begin{aligned}
Q_{\alpha}^{a}= & \left(\left\langle\left(F^{-1}\right)_{\beta}^{\gamma} G_{\gamma}^{a}\right\rangle_{0}-i \hbar\left\langle\left(F^{-1}\right)_{\beta}^{\gamma}\left\{\varphi^{a}, C_{\gamma \lambda}^{\tau}\right\}\left(F^{-1}\right)_{\sigma}^{\lambda}\right\rangle_{0} \frac{\delta \xi^{\sigma}}{\delta n^{\tau}}\right. \\
& \left.+i \hbar\left\langle\left(F^{-1}\right)_{\beta}^{\gamma}\left\{\xi^{\sigma}, \frac{\delta G_{\gamma}^{a}}{\delta n^{\lambda}}\right\}\left(F^{-1}\right)_{\sigma}^{\lambda}\right\rangle_{0}\right)\left(\left\langle F^{-1}\right\rangle_{0}^{-1}\right)_{\alpha}^{\beta} \\
= & \left(\left\langle\left(F^{-1}\right)_{\beta}^{\gamma} G_{\gamma}^{a}\right\rangle_{0}-i \hbar\left\langle\left(F^{-1}\right)_{\beta}^{\gamma} \frac{\delta}{\delta n^{\lambda}}\left(\left\{\varphi^{a}, F_{\gamma}^{\sigma}\right\}-\left\{\xi^{\sigma}, G_{\gamma}^{a}\right\}\right)\left(F^{-1}\right)_{\sigma}^{\lambda}\right\rangle_{0}\right)\left(\left\langle F^{-1}\right\rangle_{0}^{-1}\right)_{\alpha}^{\beta} .
\end{aligned}
$$

To obtain the "trivial" gauge transformations let us take

$$
T_{\alpha}^{\beta}(\varphi, \pi)=-\frac{\alpha}{2}\left(C_{\alpha \gamma}^{\beta}(\mathcal{F})\left(\gamma^{-1}\right)^{\gamma \lambda}-\left(\gamma^{-1}\right)^{\beta \gamma} C_{\alpha \gamma}^{\lambda}(\mathcal{F})\right) \pi_{\lambda}, \quad M_{\beta}^{\lambda}=\left\langle\left(F^{-1}\right)_{\beta}^{\lambda}\right\rangle_{0} .
$$

It is easy to see that due to (36) the contraction of the right-hand side of (23) with $M$ vanishes. Thus the "trivial" gauge transformations are given by

$$
\begin{aligned}
\tilde{Q}_{\alpha}^{a}= & \frac{1}{2}\left(\left\langle\left(F^{-1}\right)_{\beta}^{\gamma} G_{\gamma}^{a}\left(\xi^{\rho}-J_{(\pi)}^{\rho}\right)\right\rangle_{0}-i \hbar\left\langle\left(F^{-1}\right)_{\beta}^{\gamma} \frac{\delta}{\delta n^{\lambda}}\left(\left\{\varphi^{a}, F_{\gamma}^{\sigma}\right\}-\left\{\xi^{\sigma}, G_{\gamma}^{a}\right\}\right)\left(F^{-1}\right)_{\sigma}^{\lambda}\left(\xi^{\rho}-J_{(\pi)}^{\rho}\right)\right\rangle_{0}\right) \\
& \times\left(C_{\alpha \rho}^{\beta}(\mathcal{F})-\left(\gamma^{-1}\right)^{\beta \tau} C_{\alpha \tau}^{\delta}(\mathcal{F}) \gamma_{\delta \rho}\right) \\
= & \frac{1}{2}\left(\left\langle\left(F^{-1}\right)_{\beta}^{\gamma} G_{\gamma}^{a} \xi^{\rho}\right\rangle_{0}-i \hbar\left\langle\left(F^{-1}\right)_{\beta}^{\gamma} \frac{\delta}{\delta n^{\lambda}}\left(\left\{\varphi^{a}, F_{\gamma}^{\sigma}\right\}-\left\{\xi^{\sigma}, G_{\gamma}^{a}\right\}\right)\left(F^{-1}\right)_{\sigma}^{\lambda} \xi^{\rho}\right\rangle_{0}\right) \\
& \times\left(C_{\alpha \rho}^{\beta}-\left(\gamma^{-1}\right)^{\beta \tau} C_{\alpha \tau}^{\delta} \gamma_{\delta \rho}\right)-\frac{1}{2} Q_{\gamma}^{a}\left\langle\left(F^{-1}\right)_{\beta}^{\gamma}\right\rangle_{0}\left(C_{\alpha \rho}^{\beta}-\left(\gamma^{-1}\right)^{\beta \tau} C_{\alpha \tau}^{\delta} \gamma_{\delta \rho}\right)\left\langle\xi^{\rho}\right\rangle_{0} .
\end{aligned}
$$

In the last equality the condition $\pi_{\alpha}=\frac{1}{\alpha} \gamma_{\alpha \beta}\left(J_{(\pi)}^{\beta}-\left\langle\xi^{\beta}\right\rangle\right)=0$ was used.

The transformations induced by background fields are found from (28) to be

$$
\begin{aligned}
Q_{b}^{(\mathcal{F}) a}= & \frac{1}{\hbar}\left\langle\left\{\varphi^{a}, \Omega\right\} \bar{c}_{\beta}\left(\frac{\alpha}{2}\left(\gamma^{-1}\right)^{\beta \tau} \frac{\delta \gamma_{\tau \sigma}}{\delta \mathcal{F}^{b}}\left(\gamma^{-1}\right)^{\sigma \lambda} \pi_{\lambda}-\frac{\delta \xi^{\beta}}{\delta \mathcal{F}^{b}}\right)\right\rangle_{0} \\
= & -\left\langle\left(F^{-1}\right)_{\beta}^{\gamma} G_{\gamma}^{a}\left(\frac{1}{2}\left(\gamma^{-1}\right)^{\beta \tau} \frac{\delta \gamma_{\tau \lambda}}{\delta \mathcal{F}^{b}}\left(\xi^{\lambda}-J_{(\pi)}^{\lambda}\right)+\frac{\delta \xi^{\beta}}{\delta \mathcal{F}^{b}}\right)\right\rangle_{0} \\
& +i \hbar\left\langle\left(F^{-1}\right)_{\beta}^{\gamma} \frac{\delta}{\delta n^{\lambda}}\left(\left\{\varphi^{a}, F_{\gamma}^{\sigma}\right\}-\left\{\xi^{\sigma}, G_{\gamma}^{a}\right\}\right)\left(F^{-1}\right)_{\sigma}^{\lambda}\left(\frac{1}{2}\left(\gamma^{-1}\right)^{\beta \tau} \frac{\delta \gamma_{\tau \rho}}{\delta \mathcal{F}^{b}}\left(\xi^{\rho}-J_{(\pi)}^{\rho}\right)+\frac{\delta \xi^{\beta}}{\delta \mathcal{F}^{b}}\right)\right\rangle_{0}
\end{aligned}
$$

Let the following covariance conditions are satisfied:

$$
\begin{gathered}
\frac{\delta \gamma_{\alpha \beta}}{\delta \mathcal{F}^{a}} G_{\gamma}^{a}(\mathcal{F})=-\gamma_{\beta \lambda} C_{\gamma \alpha}^{\lambda}(\mathcal{F})-\gamma_{\alpha \lambda} C_{\gamma \beta}^{\lambda}(\mathcal{F}), \\
\frac{\delta \xi^{\alpha}}{\delta \varphi^{a}} G_{\beta}^{a}(\varphi)+\frac{\delta \xi^{\alpha}}{\delta \mathcal{F}^{a}} G_{\beta}^{a}(\mathcal{F})=C_{\beta \gamma}^{\alpha}(\mathcal{F}) \xi^{\gamma}
\end{gathered}
$$

The later is a generalization of the well known condition for the gauge fixing function for the YangMills theory. It does not look very natural, but just it gives the more or less natural expression for the symmetry transformation of the effective action. ¿From (42), (39) and (41) we have 


$$
\begin{aligned}
Q_{b}^{(\mathcal{F}) a} G_{\alpha}^{b}(\mathcal{F})= & \left\langle\left(\left(F^{-1}\right)_{\beta}^{\gamma} G_{\gamma}^{a}-i \hbar\left(F^{-1}\right)_{\beta}^{\gamma} \frac{\delta}{\delta n^{\lambda}}\left(\left\{\varphi^{a}, F_{\gamma}^{\sigma}\right\}-\left\{\xi^{\sigma}, G_{\gamma}^{a}\right\}\right)\left(F^{-1}\right)_{\sigma}^{\lambda}\right)\right. \\
& \left.\times\left(\frac{\delta \xi^{\beta}}{\delta \varphi^{b}} G_{\alpha}^{b}-\frac{1}{2}\left(C_{\alpha \rho}^{\beta}(\mathcal{F})-\left(\gamma^{-1}\right)^{\beta \tau} C_{\alpha \tau}^{\delta}(\mathcal{F}) \gamma_{\delta \rho}\right) \xi^{\rho}+\frac{1}{2}\left(\gamma^{-1}\right)^{\beta \tau} \frac{\delta \gamma_{\tau \rho}}{\delta \mathcal{F}^{b}}\left\langle\xi^{\rho}\right\rangle_{0}\right)\right\rangle_{0} \\
= & \left\langle G_{\alpha}^{a}\right\rangle_{0}-i \hbar\left\langle\frac{\delta}{\delta n^{\lambda}}\left(\left\{\varphi^{a}, F_{\alpha}^{\beta}\right\}-\left\{\xi^{\beta}, G_{\alpha}^{a}\right\}\right)\left(F^{-1}\right)_{\beta}^{\lambda}\right\rangle_{0}-\tilde{Q}_{\alpha}^{a}-Q_{\beta}^{a}\left\langle\left(F^{-1}\right)_{\gamma}^{\beta}\right\rangle_{0} C_{\alpha \lambda}^{\gamma}\left\langle\xi^{\lambda}\right\rangle_{0} .
\end{aligned}
$$

Thus if we shall choose in (31) $X_{\alpha}^{\beta}=\left\langle\left(F^{-1}\right)_{\gamma}^{\beta}\right\rangle_{0} C_{\alpha \lambda}^{\gamma}\left\langle\xi^{\lambda}\right\rangle_{0}$ and $Y_{\alpha}^{\beta}=\delta_{\alpha}^{\beta}$ then the total quantum gauge transformation reads

$$
Q_{\alpha}^{(t o t) a}=\left\langle G_{\alpha}^{a}\right\rangle_{0}-i \hbar\left\langle\frac{\delta}{\delta n^{\lambda}}\left(\left\{\varphi^{a}, F_{\alpha}^{\beta}\right\}-\left\{\xi^{\beta}, G_{\alpha}^{a}\right\}\right)\left(F^{-1}\right)_{\beta}^{\lambda}\right\rangle_{0} .
$$

In the case of the Yang-Mills theory the second term is absent, and since $G_{\alpha}^{a}$ is linear in the fields the quantum transformation is reduced to the classical one: $Q_{\alpha}^{(t o t) a}=G_{\alpha}^{a}$, i.e. $\Gamma(\varphi, \mathcal{F})$ is the classically gauge invariant effective action. In more general case the natural generalization is $Q_{\alpha}^{(t o t) a}=\left\langle G_{\alpha}^{a}\right\rangle_{0}$, that corresponds to the case of a global symmetry (14). This result can be achieved by vanishing of the second term. One needs two additional conditions on the gauge fixing function: $\frac{\delta \xi^{\alpha}}{\delta n^{\beta}}=0$ and $\left\{\xi^{\alpha}, C_{\beta \gamma}^{\lambda}\right\}=0$. They are very strong if the structure constants depend on all coordinates and momenta. As example of such theory the Ashtekar gravity can be pointed out [19]. On the other hand in the ADM gravity [20] the conditions forbid gauges on momenta only.

It may seem that the second term in (46) vanishes due to a renormalization procedure. If all operators are local the contraction of $\lambda$ and $\beta$ would result in appearance of $\delta(0)$, which in the dimensional regularization should be put zero. However the presence of the ghost propagator $\left(F^{-1}\right)_{\beta}^{\lambda}$ can give rise to nonlocality and the reasoning fails.

Finally we consider the problem of $\delta$-gauges, i.e. gauges leading to $\delta$-functions. They are obtained from (32) in the limit $\alpha \longrightarrow 0$. It is easy to see that in this limit the effective action is also invariant under the transformations (46) under the same conditions on the gauge fixing function $\xi^{\alpha}$. However

the question can arise: does the propagator $\frac{\delta^{2} W}{\delta J \delta J}$ remain non-degenerate? Note that the argument of $\delta$-function is always nonhomogeneous due to appearance of $J_{(\pi)}$. Its presence is meaningful since just it guarantees non-degeneracy. So the limit to $\delta$-gauge is well defined.

It opens new possibilities for simplification of the quantum gauge transformations. Using $\delta$-gauges one can make the ghost propagator $F_{\alpha}^{\beta}$ independent on fields. Then provided the second term in (39) vanishes we come to $Q_{\alpha}^{a}=\left\langle G_{\alpha}^{a}\right\rangle_{0}$, that gives the classical symmetry for the Yang-Mills theory without use of background fields. In this case the result can be achieved with help of such noncovariant gauges as $\xi=A_{3}$ or $\xi=A_{0}$ (here $A_{\mu}$ is the gauge potential).

\section{CONCLUSIONS AND DISCUSSION}

In this article we have considered symmetry properties of the effective action for general gauge theories. By taking as a basis the Hamiltonian BRST formalism and the path integral quantization we have found the quantum gauge transformations in terms of quantum averages. Moreover there are additional symmetry transformations of the effective action: the "trivial" symmetries, which vanish on mass shell, and the gauge transformations induced by background fields. (We don't identify background fields with average fields so that the effective action is a functional of these two variables.) Finally we have shown that combining all these symmetries one can obtain more simple form of the transformations. For example, for the rank one theory under some conditions on the gauge fixing function the gauge transformations are represented as an average of the classical ones. For YangMills theory, in which the gauge transformations are linear in the fields, it gives the classical result for the quantum symmetry also.

Unfortunately this generalization for the rank one theory is very weak because of the covariance conditions (44). Their solution is a problem and, may be, the result isn't worth the efforts. As we 
have seen this is only the attempt to find some simple form of the symmetry, so there is no need to introduce new difficulties. On the other hand this formalism translates the problem of search for the (classically) invariant effective action to the problem of solving of equations.

We did not concern of the renormalization problem. It becomes complicated due to that composite operators enter into expressions for the quantum gauge transformations. However this is the standard difficulty for investigations which deal with the Ward identities. So we suppose that in real calculations all expressions should be renormilized taking into account a mixing of operators.

Of course, such calculations should be carried out with help of expansion in $\hbar$. Each order together with previous ones gives restrictions to the corresponding order of expansion of the effective action. It is worth to notice that the first nontrivial correction to the classical transformations is fully defined by the classical action since it is proportional $\sim \hbar \frac{\delta^{2} W}{\delta J \delta J} \sim \hbar\left(\frac{\delta^{2} \Gamma}{\delta \psi \delta \psi}\right)^{-1} \sim \hbar\left(\frac{\delta^{2} S}{\delta \psi \delta \psi}\right)^{-1}$.

The consideration of this article can be translated to the Lagrangian BV formalism. The proof of its equivalence to the Hamiltonian BFV formalism can be found for instance in 21]. Within this framework all symmetry properties of the effective action are contained in the Zinn-Justin equation 22] $(\Gamma, \Gamma)=0$, where $($,$) is the antibracket and \Gamma$ is a functional of fields and antifields [11,14, 15. Since it has the same form as the classical master equation $(S, S)=0$ one can define a BRST structure associated with $\Gamma$ [23]. However there are small difficulties similar to difficulties in the Hamiltonian approach. Whereas for the proper solution $S$ ghost number considerations allow to conclude that $S_{0}$ is the local invariant classical action, we cannot maintain this for $\Gamma_{0}$. (Here the subscript 0 means vanishing all ghosts and antifields.) This is bacause in the quantum case to fix the gauge we must introduce auxilary fields. The antifield $\bar{C}^{\alpha *}$ for one of them has ghost number zero 14. So $\Gamma_{0}$ will be local invariant only on the surface $\left(\frac{\partial \Gamma}{\partial C^{\alpha *}}\right)_{0}=0$. It should be viewed as an equation on the auxilary field $\bar{\pi}_{\alpha}$ which plays a role of momentum conjugated to Lagrange multiplier and has ghost number zero. This corresponds to necessity of removal all auxilary fields and gives the concret way for this. Our choice of the Hamiltonian formalism is connected to wish to avoid the problem of solution of the quantum master equation [11, 14, 15]. However notice that in BV approach the quantum algebra may be simpler then in our case [8, 13].

Another progress can be connected with an application of quantum groups. It is just the structure that should manage the quantum symmetry that is a deformation of the classical one.

\section{ACKNOWLEDGMENTS}

The author is very grateful to D.V.Vassilevich and V.A.Franke for helpful and valuable discussions.

[1] 't Hooft G., Nucl. Phys. B33, 173 (1971), B35, 167 (1971).

[2] B.S.DeWitt, in: Quantum Gravity 2, eds. C.J. Isham, R.Penrose and D.W.Sciama. (Clarendon Press, Oxford, 1981).

[3] L.F.Abbott, Nucl. Phys. B185, 189 (1981);

D.G.Boulware, Phys. Rev. D 23, 389 (1981);

C.F.Hart, Phys. Rev. D 28, 1993 (1983).

[4] G.A. Vilkovisky, in: Quantum Theory of Gravity, ed. S.M. Christensen (Hilger, Bristol 1984).

[5] G.A. Vilkovisky, Nucl. Phys. B234, 127 (1984).

[6] B.S.DeWitt, in: Quantum Field Theory and Quantum Statistics - Essays in Honour of the Sixtieth Birthday of E.S.Fradkin, eds. I.A.Batalin, C.J.Isham and G.A.Vilkovisky, (Hilger, Bristol, 1987).

[7] P.M.Lavrov, Mod. Phys. Let. 6A, 2051 (1991);

P.M.Lavrov, Phys. Lett. B 366, 160 (1996), hep-th/9507105 preprint. 
[8] S.Falkenberg, B.Geyer, P.Lavrov and P.Moshin, Int. J. Mod. Phys. A13, 4077 (1998), hep-th/9710050 preprint.

[9] C.Becchi, A.Rouet and R.Stora, Comm. Math. Phys. 42, 127 (1975); Ann. Phys. 98, 287 (1976); I.V.Tyutin, Lebedev preprint FIAN No.39 (1975).

[10] E.S. Fradkin and G.A. Vilkovisky, Phys. Lett. B 55, 244 (1975);

I.A. Batalin and G.A. Vilkovisky, Phys. Lett. B 69, 309 (1977);

E.S. Fradkin and T.E. Fradkina, Phys. Lett. B 72, 343 (1978).

[11] I.A.Batalin and G.A.Vilkovisky, Phys. Lett. B 102, 27 (1981); Phys. Rev. D 282567 (1983).

[12] B.L.Voronov, P.M.Lavrov and I.V.Tyutin, Sov. J. Nucl. Phys. 36, 292 (1982).

[13] A.Sen, B.Zwiebach, Phys. Lett. B 320, 29 (1994), hep-th/9309027 preprint;

M.A.Grigoriev, A.M.Semikhatov and I.Yu.Tipunin, hep-th/9804156 preprint.

[14] J.Gomis, J.Paris and S.Samuel, Phys. Rep. 259, 1 (1995), hep-th/9412228 preprint.

[15] M.Henneaux and C.Teitelboim, Quantization of Gauge Systems (Princeton University Press, Princeton, 1992).

[16] B.S.DeWitt, Dynamical Theory of Groups and Fields (Gordon and Breach, New York, 1965).

[17] M. Henneaux, Phys. Rep. 126, 1 (1985).

[18] D.Anselmi, Class. Quant. Grav. 11, 2481 (1994), hep-th/9309085 preprint.

[19] A.Ashtekar, Phys. Rev. Lett. 57, 2244 (1986); Phys. Rev. D 36, 1587 (1987).

[20] R.Arnowitt, S.Deser and C.W.Misner, in: Gravitation: An Introduction to Current Research, ed. L.Witten, (Wiley, New York, 1962).

[21] C.Batlle, J.Gomis, J.Paris and J.Roca, Nucl. Phys. B329, 139 (1990);

A.Dresse, J.M.L.Fisch, P.Gregoire and M.Henneaux, Nucl. Phys. B354 191 (1991);

G.V.Grigorian, R.P.Grigorian and I.V.Tyutin, Sov. J. Nucl. Phys. 531058 (1991); Nucl. Phys. B379, 304 (1992).

[22] J.Zinn-Justin, Renormalization of Gauge Theories, in Trends in Elementary Particle Theory, edited by H.Rollnik and K.Dietz, Lecture Notes in Physics, Vol 37, (Springer-Verlag, Berlin, 1975).

[23] J.Gomis and J.Paris, Nucl. Phys. B431, 378 (1994), hep-th/9401161 preprint. 